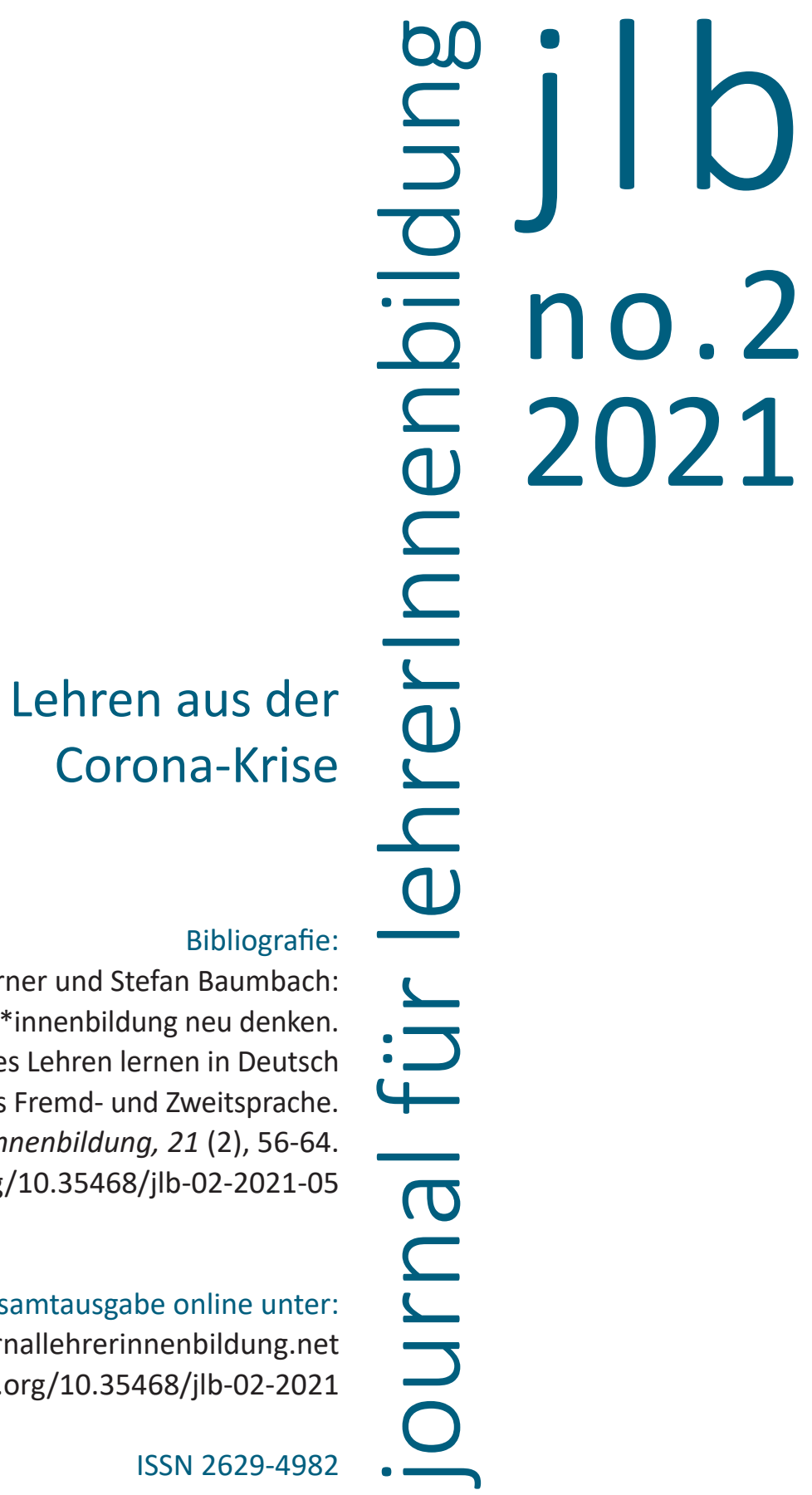


05

Theres Werner und Stefan Baumbach

\section{Lehrer*innenbildung neu denken. Digitales Lehren lernen in Deutsch als Fremd- und Zweitsprache}


Dass die „digitale Kompetenz [...] eine Schlüsselkompetenz des 21. Jahrhunderts" (DigCompEdu, 2017, S. 1) ist, wurde mit der CoronaPandemie seit März 2020 zunehmend deutlich. In kürzester Zeit mussten Lehr-Lernsettings auf digitale Formate umgestellt werden. Das bisherige Verständnis von Lernen muss hinterfragt und kann neu gedacht werden, sodass Raum für neue Formate entsteht. Um der gegenwärtigen Situation mit den Bedingungen des Distance Learnings Rechnung zu tragen, entstand das Projekt „Digitales Lehren lernen in Deutsch als Fremd- und Zweitsprache" (DiLele DaF/DaZ) ${ }^{1}$ an der Friedrich-SchillerUniversität Jena. Dafür wurde ein Seminarkonzept entwickelt, in dem Lehramtsstudierende entsprechend ihres Vorwissens in einem geschützten Raum verschiedene Formate des digitalen Lehrens und Lernens erproben und reflektieren. Digitales Lernen ist neben dem Lerninhalt gleichzeitig Lernmethode und Lernprodukt. Ziel ist es, einerseits die Studierenden auf ihren Unterrichtsalltag praxisnah vorzubereiten und andererseits diese Lehr-Lernform in die Lehrpersonenbildung des Fachbereiches Deutsch als Fremd- und Zweitsprache (DaF/DaZ) curricular einzubinden und systematisch weiterzuentwickeln.

\section{Ausgangsbedingungen auf bildungspolitischer Ebene}

Mit dem Blick auf die Ausbildung von Lehrkräften ist erkennbar, dass seit 2017 auf bildungspolitischer Ebene bereits vor der Corona-Krise äußere Rahmenbedingungen zur Stärkung digitaler Kompetenzen von Lehrkräften geschaffen wurden. Mit der Strategie der Kultusministerkonferenz (KMK) zur Bildung in der digitalen Welt (KMK, 2017) und der des Landes Thüringen zur Digitalisierung im Hochschulbereich (Thüringer Strategie, 2017) sowie den Empfehlungen zur Digitalisierung in der Hochschullehre (KMK, 2019) werden konkrete Ziele angestrebt, Hochschullehre zu digitalisieren, um Studierende auf die zukünftige Arbeitswelt vorzubereiten. Auch die vom Bundesministerium für Bildung und Forschung (BMBF) geförderte Qualitätsoffensive Lehrer-

1 Das Projekt wird mit einem Fellowship im Rahmen der Programmlinie Innovationen in der digitalen Hochschullehre Thüringen durch den Stifterverband und das Thüringer Ministerium für Wirtschaft, Wissenschaft und Digitale Gesellschaft gefördert (Stifterverband, o. J.). 
bildung mit dem Schwerpunkt Digitalisierung in der Lehrerbildung (BMBF, 2018) unterstützt solche Vorhaben. Nicht zuletzt wird mit dem Thüringer Lehrerbildungsgesetz das Ziel verfolgt, Lehrkräfte so zu qualifizieren, dass sie "am Prozess einer innovativen Schulentwicklung“ (ThürLbG, §2 Abs.1) mitwirken können. Zweifelsfrei gehört digitales Lehren und Lernen mit zu solchen Innovationen und sollte sich in den Curricula der Lehrpersonenbildung wiederfinden.

\section{Ausgangsbedingungen auf der praxisbezogenen Ebene im Schulkontext}

Wird der Fokus auf die Praxis mit der aktuellen Situation des Fernunterrichts gerichtet (siehe dazu auch Fickermann \& Edelstein, 2020), zeichnet sich ein unverkennbarer Bedarf ab, Lehrkräfte in diesem Bereich verstärkt und strukturiert zu qualifizieren.

Aus Elternperspektive wird dies durch die Befragung zum Digitalen Lernen während der Corona-Krise deutlich. Neben dem Bedarf, den geschulten Umgang mit Lernmanagementsystemen bei Lehrenden und Lernenden zu fördern, müsse auch die Lernprozessbegleitung beim digitalen Lernen weiterentwickelt werden (Bildung Jena, 2020, S. 8ff.). Das bestätigen auch die Ergebnisse der bundesweiten Elternbefragung, aus der hervorgeht, dass mehrheitlich der Wunsch nach mehr Struktur im Lernprozess bestehe (Wildemann \& Hosenfeld, 2020, S. 29).

Aus Lehrpersonenperspektive liege mit $69 \%$ das größte Verbesserungspotenzial im Ausbau eigener digitaler Kompetenzen (Forsa, 2020, S. 23). Dass die Bereitschaft bei Lehrenden zur Weiterentwicklung in diesem Bereich gegeben sei, zeigt die Studie von Dreer und Kracke (2020, S. 3).

Zusätzliche Herausforderungen zu den aufgeführten Bedarfen bestehen im Kontext von DaF/DaZ insbesondere bei neu zugewanderten Lernenden. Denn auch im Fernunterricht müsse neben einer sprachlichen Förderung und der Verknüpfung von fachlichem und sprachlichem Lernen auch die sprachliche Unterstützung sowohl der Schüler*innen als auch der Eltern bei der Lernbegleitung berücksichtigt werden (Gogolin, 2020, S. 179f.). 


\section{Ausgangsbedingungen auf hochschuldidaktischer Ebene}

In der akademischen Lehre wurden vor dem Hintergrund der Corona-Pandemie, zumindest im Sommersemester 2020, die Lehrveranstaltungen als "Notfall“-Reaktion (Reinmann, 2020, S. 2) auf politische Entscheidungen ad hoc in digitale Lehrangebote geändert, um den Lehrbetrieb aufrecht zu erhalten. Um nicht nur vorübergehende Lösungen als Ersatz im Sinne eines Emergency Remote Teachings (Hodges, Moore, Lockee, Trust \& Bond, 2020, o. S.) zu finden, sondern auch nachhaltige Lehren aus der Corona-Krise zu ziehen und den zukünftigen Generationen eine Teilhabe an der digitalen Bildungswelt zu ermöglichen, bedarf es mehrerer Schritte. Erstens besteht die Notwendigkeit, evidenzbasierte Modelle aus dem Bereich des online Lernens heranzuziehen (vgl. Bauer, Hafer, Hofhues, Schiefner-Rohs, Thillosen, Volk \& Wannemacher, 2020; Hafer, Mauch \& Schumann, 2019; Hofhues, Schiefner-Rohs, Aßmann \& Brahm, 2020). Zweitens sollte die Modellfunktion der Hochschullehre für die Lehrpersonenbildung bedacht werden, denn „Hochschulen sind als Lernorte zur Professionalisierung [...] prädestiniert" (Legutke \& Schart, 2016, S. 36). Drittens sind die Bedürfnisse der Lernenden einzubeziehen, die bisher zu wenig Berücksichtigung fanden (Dittler \& Kreidl, 2020, S. 44f.; Holmes, Anastopoulou, Schaumburg \& Mavrikis, 2018). Viertens steht die Lehrkräftebildung vor der Herausforderung, dass der Schulunterricht nur begrenzt planbar ist, da er situationsabhängig durch Unvorhersehbarkeiten geprägt ist (vgl. Kunter, Baumert, Blum, Klusmann, Krauss \& Neubrand, 2011, S. 30). Daher sind in der akademischen Lehrpersonenbildung geschützte Räume notwendig, in denen in Szenarien das Lehrer*innenhandeln in digitalen Lehr-Lernarrangements unter Peers erprobt werden kann. 


\section{Seminarkonzeption}

Mit den oben aufgeführten Ausgangsbedingungen ergeben sich Ziele, die mit der Seminarkonzeption verfolgt werden:

a) die Aneignung von digitalen Lerninhalten unter Verwendung digitaler Lernmethoden

b) Berücksichtigung der heterogenen Lernvoraussetzungen der Studierenden in den Lernzielen

c) als Lernergebnis und Prüfungsleistung ein digitales Lernprodukt

Für die Lerninhalte und -methoden (a) werden digitale Selbstlernbausteine entwickelt, die sich thematisch an fachwissenschaftlichen Grundlagen zum digitalen Lernen und an den Ergebnissen der oben aufgeführten Studien orientieren. Sie beinhalten beispielsweise Grundlagen des methodisch-didaktischen digitalen Lehrens und Lernens unter Berücksichtigung synchroner und asynchroner Formate mit der Verschränkung von Online- und Präsenzunterricht, Mediennutzung digitaler Tools und deren didaktisches Potenzial, medienkritischer Umgang, Datenschutz und Netiquette, Lernprozessbegleitung, heterogenitätssensibles Unterrichten, Kooperation mit Eltern, aber auch digitalbedingte Stressbewältigung durch Achtsamkeit.

Zur Berücksichtigung der heterogenen Lernziele (b) wird das problemorientierte Lernen (POL) (Weber, 2017) eingesetzt, um dem unterschiedlichen (fremdsprachen-)didaktischen Vorwissen, Vorerfahrungen, Motivationen sowie Lernvoraussetzungen Studierender gerecht zu werden. Das POL-Szenario, aus der schulischen Unterrichtspraxis stammend, wird für den akademischen Kontext didaktisiert und ermöglicht den Studierenden neben selbstgesteuertem und kooperativem Lernen auch eine Wissensaneignung ausgerichtet auf Anwendung und Praxistransfer. So sind Selbsterfahrungen mit dieser Lernform möglich, welche den Perspektivwechsel in die Schüler*innenrolle zukünftig erleichtern können. Gerahmt durch das Problemszenario und ausgehend von ihrem diagnostizierten Kenntnisstand zur eigenen digitalen Kompetenz mit Hilfe des DigCompEdu-Tools (DigCompEdu-Tool, o. J.) legen die Studierenden selbstständig ihre Lernziele für die Lehrveranstaltung fest. Die Lernziele orientieren sich an der Lernzieltaxonomie mit den Stufen 1. remember, 2. understand, 3. apply, 4. analyze, 5. evaluate und 6. create, um das eigene Lernen im Sinne eines konstruktivistischen Verständnisses zu strukturieren und nachzuvollziehen (Anderson et al., 2014, p. 28). 
Die Prüfungsleistung ist mit dem Lernergebnis abgestimmt (c) und setzt sich aus einem individuellen (50\%) und einem kollektiven Teil (50\%) zusammen. Die individuelle Teilleistung umfasst erstens die Reflexion des Gelernten und der erreichten Lernziele und zweitens das Feedback zu einem Lernprodukt der Kommiliton*innen anhand einer Bewertungsmatrix. Die kollektive Teilleistung erfolgt mit dem Erstellen eines digitalen Lernprodukts, welches in Partner-, Gruppen- oder auch Einzelarbeit erfolgen kann.

Um die Kohärenz zwischen a) Lernmethoden, b) Lernzielen und c) Prüfungsleistungen herzustellen, wird der Lehrveranstaltung das Konzept des Constructive Alignments zu Grunde gelegt (Bigg, 1996) und alle drei Bereiche aufeinander abgestimmt, indem erstens in verschiedenen digitalen Selbstlernbausteinen in unterschiedlichen Formaten unter der Berücksichtigung individueller Lernziele Wissen angeeignet wird ( $a$ und $b$ ). Zweitens wird die eigene Lernentwicklung anhand der individuellen Lernziele mit Hilfe von Prompts formativ reflektiert und soll als ein erster Prüfungsteil abgeleistet werden (b und c). Und drittens werden die Lehr-Lernmethoden auf die Prüfungsleistung abgestimmt, indem digitale Lernprodukte über das angeeignete Wissen als zweiter Prüfungsteil erbracht werden ( $a$ und $c$ ).

\section{Ausblick}

Mit dem entwickelten Seminarkonzept, welches im Sommersemester 2021 eingesetzt und evaluiert wird, wird perspektivisch neben einer nachhaltigen Nutzung der Lernergebnisse der Studierenden auch ein Transfer in die zweite und dritte Phase der Lehrpersonenbildung angestrebt. Die digitalen Lernprodukte können für nachfolgende Lehrveranstaltungen erstens als Beispiele und zweitens mit entsprechender Qualität zukünftig als Teil eines Selbstlernbausteins verwendet werden. Drittens können sie von den Studierenden selbst in den anderen studierten Fächern und Fachdidaktiken und viertens als Selbstlernbausteine in der Weiterbildung von Lehrpersonen genutzt werden. Beispielsweise von berufsbegleitenden Studierenden, die als Multiplikator*innen in ihrer Schule fungieren und diese zur Fortbildung im eigenen Kollegium einsetzen. 


\section{Literatur}

Anderson, L. W., Krathwohl, D. R., Airasian, P. W., Cruikshank, K. A., Myer, R. E., Pintrich, P. R., Raths, J. \& Wittrock, M. C. (2014). A Taxonomy for Learning, Teaching, and Assessing: A Revision of Bloom's. Harlow: Pearson Education.

Bauer, R., Hafer, J., Hofhues, S., Schiefner-Rohs, M., Thillosen, A., Volk, B. \& Wannemacher, K. (Hrsg.). (2020). Vom E-Learning zur Digitalisierung. Mythen, Realitäten, Perspektiven. Münster u. a.: Waxmann.

Bigg, J. (1996). Enhancing teaching through constructive alignment. Higher Education 32, 347-364.

Bildung Jena (2020). Ergebnisse der Befragung Organisation von Digitalem Lernen während der Corona-Krise aus Elternsicht. Verfügbar unter https://bildung.jena. de/de/ergebnisse-befragung-organisation-von-digitalem-lernen-waehrend-dercorona-krise-aus-elternsicht [23.12.2020].

BMBF (2018). Richtlinie zur Förderung von Projekten in der Qualitätsoffensive Lehrerbildung mit den Schwerpunkten Digitalisierung in der Lehrerbildung. Verfügbar unter https://www.bmbf.de/foerderungen/bekanntmachung-2097.html [23.12.2020].

DigCompEdu (2017). Broschüre zur digitalen Kompetenz Lehrender. Verfügbar unter https://ec.europa.eu/jrc/sites/jrcsh/files/digcompedu_leaflet_de-2018-09-21 pdf.pdf [23.12.2020].

DigCompEdu-Tool (o. J.). Tool zur Einschätzung der digitalen Kompetenz von Lehrkräften an allgemein- oder berufsbildenden Schulen. Verfügbar unter https:// ec.europa.eu/eusurvey/runner/DigCompEdu-S-DE [23.12.2020].

Dittler, U. \& Kreidl, C. (2020). Vom Mythos zur Realität: Lernenden-zentrierte Überlegungen zur Digitalisierung. In R. Bauer, J. Hafer, S. Hofhues, M. Schiefner-Rohs, A. Thillosen, B. Volk \& K. Wannemacher (Hrsg.), Vom E-Learning zur Digitalisierung. Mythen, Realitäten, Perspektiven (S. 40-54). Münster u. a.: Waxmann.

Dreer, B. \& Kracke, B. (2020). Befragung von Thüringer Lehrer*innen während der durch die Corona-Krise bedingten Schulschließungen 2020 - Bericht erster Ergebnisse. Verfügbar unter https://www.uni-jena.de/unijenamedia/Thueringer_Studie_zum_ Unterricht_in_der_Corona krise.pdf [23.12.2020].

Fickermann, D. \& B. Edelstein (2020). Editorial. „Langsam vermisse ich die Schule ...“. Schule während und nach der Corona-Pandemie. DDS Die deutsche Schule. Zeitschrift für Erziehungswissenschaft, Bildungspolitik und pädagogische Praxis, Beiheft 16, 9-36. https://doi.org/10.31244/9783830992318.

Forsa (2020). Das Deutsche Schulbarometer Spezial Corona-Krise. Ergebnisse einer Befragung von Lehrerinnen und Lehrern an allgemeinbildenden Schulen im Auftrag der Robert Bosch Stiftung in Kooperation mit der ZEIT. Verfügbar unter https://www.schulwerk-bayern.de/fileadmin/pics/Aktuelles_2020/20-04-27_ Schulentwicklung_Forsat.pdf [23.12.2020].

Gogolin, I. (2020). Sprachliche Förderung, sprachliche Bildung und Lernen im Deutschen als Zweitsprache während und nach der Pandemie. DDS Die deutsche Schule. Zeitschrift für Erziehungswissenschaft, Bildungspolitik und pädagogische Praxis, Beiheft 16, 175-188. https://doi.org/10.31244/9783830992318.

Hafer, J., Mauch, M. \& Schumann, M. (Hrsg.). (2019). Teilhabe in der digitalen Bildungswelt. Münster u. a.: Waxmann.

Hodges, C., Moore, S., Lockee, B., Trust, T. \& Bond, A. (2020). The difference between emergency remote teaching and online learning. Educause Review. Verfügbar 
unter articles/2020/3/the-difference-between-emergency-remote-teaching-andonline-learning [23.12.2020].

Hofhues, S., Schiefner-Rohs, M., Aßmann, S. \& Brahm, T. (Hrsg.). (2020). Studierende Medien - Universität. Einblicke in studentische Medienwelten. Münster u. a.: Waxmann.

Holmes, W., Anastopoulou S., Schaumburg, H. \& Mavrikis, M. (2018). Personalisiertes Lernen mit digitalen Medien. Ein roter Faden. Stuttgart: Robert Bosch Stiftung.

KMK (2017). Bildung in der digitalen Welt. Strategie der Kultusministerkonferenz. Verfügbar unter https://www.kmk.org/fileadmin/Dateien/pdf/PresseUndAktuelles/ 2017/Strategie_neu_2017_datum_1.pdf [23.12.2020].

KMK (2019). Empfehlungen zur Digitalisierung in der Hochschullehre. Verfügbar unter https://www.kmk.org/fileadmin/Dateien/pdf/PresseUndAktuelles/2019/BS_ 190314_Empfehlungen_Digitalisierung_Hochschullehre.pdf [23.12.2020].

Kunter, M., Baumert, J., Blum, W., Klusmann, U., Krauss, S. \& Neubrand, M. (Hrsg.). (2011). Professionelle Kompetenz von Lehrkräften. Ergebnisse des Forschungsprogramms COACTIV. Münster u. a.: Waxmann.

Legutke, M. K. \& Schart, M. (2016). Fremdsprachliche Lehrerbildungsforschung: Bilanz und Perspektiven. In M. K. Legutke \& M. Schart (Hrsg.), Fremdsprachendidaktische Professionsforschung: Brennpunkt Lehrerbildung. (S. 9-46). Tübingen: Narr Francke Attempto.

Reinmann, G. (2020). Universitäre Lehre in einer Pandemie - und danach?. Impact Free, 29. Verfügbar unter https://gabi-reinmann.de/wp-content/uploads/2020/06/ Impact_Free_29.pdf [23.12.2020].

Stifterverband (o. J.). Fellowship für Innovationen in der digitalen Hochschullehre Thüringen. Gefördert durch den Stifterverband und das Thüringer Ministerium für Wirtschaft, Wissenschaft und Digitale Gesellschaft. Verfügbar unter https://www. stifterverband.org/digital-lehrfellows-thueringen/2020/werner [23.12.2020].

Thüringer Strategie (2017). Thüringer Strategie zur Digitalisierung im Hochschulbereich der Thüringer Landesrektorenkonferenz und dem Thüringer Ministerium für Wirtschaft, Wissenschaft und Digitale Gesellschaft. Verfügbar unter https://www. tlpk.de/fileadmin/Downloads/Allgemeines/20171215_Digitalisierungsstrategie_ Thuer_Hochschulen_signed.pdf [23.12.2020].

ThürLbG (2008). Thüringer Lehrerbildungsgesetz. Verfügbar unter https://landes recht.thueringen.de/bsth/document/jlr-LehrBiGTHpG1 [23.12.2020].

Weber, A. (2017). Problem-Based Learning. Ein Handbuch für die Ausbildung auf der Sekundarstufe II und der Tertiärstufe (2. Aufl.). Bern: h.e.p.

Wildemann, A. \& Hosenfeld, I. (2020). Bundesweite Elternbefragung zu Homeschooling während der Covid 19-Pandemie. Erkenntnisse zur Umsetzung des Homeschoolings in Deutschland. Verfügbar unter https://homeschoolingstudie2020. zepf.eu [23.12.2020]. 


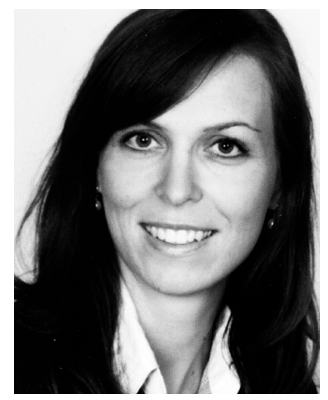

Theres Werner, wiss. Mitarbeiterin am Institut für $\mathrm{DaF} / \mathrm{Z}$ und Interkulturelle Studien, Friedrich-Schiller-Universität Jena.

Arbeitsschwerpunkte:

Professionalisierung in der DaF/Z-Lehrkräfteausund -weiterbildung

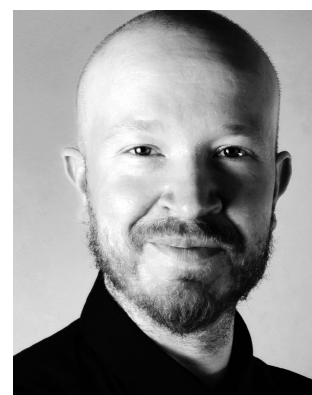

theres.werner@uni-jena.de

Stefan Baumbach, M. A., Fachleiter für DaZ und Lehrbeauftragter am Institut für DaF/Z und Interkulturelle Studien, Friedrich-Schiller-Universität Jena.

Arbeitsschwerpunkte:

Lehrkräftebildung und Professionsforschung in $\mathrm{DaF} / \mathrm{Z}$, Auslandsschulwesen

stefan.baumbach@uni-jena.de 